\title{
Compound gravitational lensing as a probe of dark matter substructure within galaxy halos
}

\author{
R. Benton Metcalf \\ Institute of Astronomy, University of Cambridge, Cambridge CB3 OHA, UK \\ and \\ Piero Madau \\ Department of Astronomy and Astrophysics, University of California, Santa Cruz, CA 95064
}

\begin{abstract}
We show how observations of multiply-imaged quasars at high redshift can be used as a probe of dark matter clumps (subhalos with masses $\lesssim 10^{9} \mathrm{M}_{\odot}$ ) within the virialized extent of more massive lensing halos. A large abundance of such satellites is predicted by numerical simulations of galaxy formation in cold dark matter (CDM) cosmogonies. Small-scale structure within galaxy halos affects the flux ratios of the images without appreciably changing their positions. We use numerical simulations to quantify the effect of dark matter substructure on the distribution of magnifications, and find that the magnification ratio of a typical image pair will deviate significantly from the value predicted by a smooth lensing potential if, near the Einstein radius, only a few percent of the lens surface density is contained in subhalos. The angular size of the continuum source dictates the range of subclump masses that can have a detectable effect: to avoid confusion with gravitational microlensing caused by stars in the lens galaxy, the background source must be larger than the optical continuum-emitting region of a QSO. We also find that substructure will cause distortions to images on milli-arcsecond scales and bias the distribution of QSO magnification ratios - two other possible methods of detection.
\end{abstract}

Subject headings: cosmology: theory - dark matter - galaxies: formation - gravitational lensing

\section{Introduction}

The popular model of hierarchical structure formation in a universe dominated by cold dark matter $(\mathrm{CDM})$, while quite successful in matching the observed large-scale density distribution, is currently facing a 'small-scale crisis' (e.g., Moore 2001). First and foremost, CDM simulations appear to produce halos that are too centrally concentrated compared to the mass distribution inferred from the rotation curves of (dark matter-dominated) dwarf galaxies (Moore et al. 1999b; Klypin et al. 2001; Navarro, Frenk, \& White 1997). In addition to the cuspy density profiles, the predicted abundance of satellites of mass greater than $10^{8} \mathrm{M}_{\odot}$ within the virialized extent of a Milky Way's halo is more than an order of magnitude larger than the number of dwarf galaxies with comparable mass observed within the Local Group (Moore et al. 1999a; Klypin et al. 1999b; Mateo 1998). A number of related but more complex problems is also being discussed in the literature (see Sellwood \& Kosowsky 2001 for a recent review), together with the significance of some of the observations (van den Bosch \& Swaters 2001). It is unclear at this stage whether the CDM paradigm is actually incorrect and needs to be modified to include, e.g., self-interacting (Spergel \& Steinhardt 2000) or warm dark matter (Bode, Ostriker, \& Turok 2000; Colin, Avila-Reese, \& Valenzuela 2000), or whether some 
of these discrepancies may have a more 'astrophysical' origin. Feedback processes such as photoionization, for example, may prevent gas cooling and inhibit star formation in the majority of the dwarf subhalos (e.g. Bullock, Kravtsov, \& Weinberg 2001). In this case the dark matter satellites of the Milky Way would still be present, but only a small fraction of them would actually be visible, having formed stars prior to the reheating of the intergalactic medium.

Gravitational lensing is a powerful tool for studying the structure of dark matter halos. The statistics of wide-separation lenses can be used to constrain the amount of mass in the cores of dark matter halos on group and cluster mass scales (Keeton \& Madau 2001; Li \& Ostriker 2001; Flores \& Primack 1996). Similarly, substructure in galaxy halos can be probed by gravitational lensing even if the satellites contain too few baryons to be observable. A lump approximating a singular isothermal sphere (SIS) of one-dimensional velocity dispersion $\sigma=10 \mathrm{~km} \mathrm{~s}^{-1}$ (corresponding to a mass of order $10^{8} \mathrm{M}_{\odot}$ ) produces a deflection angle of about 3 milli-arcsecond if it acts as a lone lens. If the lump resides in a larger halo this deflection will effectively be magnified. This can cause distortions of images on ten milli-arcsecond scales and appreciable changes to the total flux.

It has been pointed out by Mao \& Schneider (1998) that substructure in the lens galaxy can explain the discrepancy between the observed and ('simple') model-predicted flux ratios in the quadruply-imaged QSO B1422+231. These authors considered two illustrative pictures of substructure, point-like "globular clusters" with masses of $10^{6} \mathrm{M}_{\odot}$ and a plane-wave smooth fluctuation. In this paper we study how to use gravitationally lensed QSOs as a probe of the existence of dark matter subclumps within the lensing potential, the small-scale structure that is indeed predicted by numerical CDM simulations. The format is as follows. In $\S 2$ we discuss the survival of subclumps in dark matter halos. A model for halo substructure is presented in $\S 3$ where it is argued that many subclumps will survive to within a few kpc of a galactic halo's center. The formalism for describing lensings by subclumps is introduced in $\S$, and the numerical simulations used to study the magnification and image distribution are described in $\S 5$. Specific examples of lensing systems and subclump distributions are investigated in $\S 6$. Finally, we discuss the implications of this work in $\S$.

\section{Survival of substructure in galaxy halos}

In hierarchical cosmogonies - of which CDM is the best studied example - all the mass of the universe at early times is contained in small-mass halos that later merge into larger and larger systems. The problem of how "halos orbiting within halos" evolve with time is rather complex (Johnston, Spergel, \& Hernquist 1995; Moore, Katz, \& Lake 1996; Klypin et al. 1999a). Survival of satellites in galaxy clusters has been investigated by a number of authors (see Tormen, Diaferio, \& Syer 1998; Ghigna et al. 1998) where it has been found that somewhere between $10 \%$ and $20 \%$ of the cluster mass remains in bound subclumps. Simulating substructure in galaxy halos is much more computationally demanding. Very high resolution cosmological Nbody simulations (Klypin et al. 1999b; Moore et al. 1999a) and semi-analytic modeling (Bullock, Kravtsov, \& Weinberg 2000) have shown that a large number of subclumps do survive in CDM theories, and that galaxy halos resemble scaled down versions of galaxy clusters. According to these simulations, the mass function of subclumps is approximately $d n / d m \propto m^{-2}$ down to a typical resolved mass of $\gtrsim 10^{7} \mathrm{M}_{\odot}$.

To understand the destruction process of dark matter clumps and estimate the range of substructure masses and radii that are to be expected, we can use some simple analytic arguments. Dynamical friction will cause only the most massive satellites, $m \gtrsim 10^{10} \mathrm{M}_{\odot}$, to sink into the center of galaxy size halos. For 
the range of (smaller) masses we are interested in, the most important factor in the evolution of substructure is tidal stripping within the parent halo that they inhabit. The tidal radius $r_{t}$ of a clump of bound mass $m$ can be estimated as

$$
r_{t} \simeq R\left[\frac{m}{3 M(R)}\right]^{1 / 3}
$$

where $R$ is measured from the center of the (host) halo, and $M(R)$ is the halo mass within that radius (Binney \& Tremaine 1987). This constraint on the subclump size is plotted in Figure 1 for a Milky Way-like galaxy halo approximating a SIS mass density profile,

$$
\rho(r)=\frac{\sigma_{\text {halo }}^{2}}{2 \pi G r^{2}}
$$

with circular velocity $V_{c}=\sqrt{2} \sigma_{\text {halo }}=226 \mathrm{~km} \mathrm{~s}^{-1}$, and for two different radii $R$. Any clump of radius $r>r_{t}$ will be stripped of mass as it falls towards the center. By losing mass and shrinking in size the clump may eventually become quasistable when it reaches approximately the tidal stripping line evaluated at the pericenter of its orbit (dynamical friction will eventually drag them in on a long timescale and gravitational heating caused by the time varying tidal force will slowly pump energy into them). Four such tracks are shown in Figure 1 under the assumption that the clump density profile remains unaffected by mass stripping.

SIS subclumps will lose a large fraction of their mass before they can reach the solar radius, $R \simeq 10 \mathrm{kpc}$. A clump with a more realistic NFW profile (Navarro, Frenk, \& White 1997),

$$
\rho(r)=\frac{\rho_{c} \delta_{c}}{\left(r / r_{s}\right)\left(1+r / r_{s}\right)^{2}},
$$

is more centrally concentrated and can reduce its size without loosing much of its mass. This is only true until $r_{t}(R) \lesssim r_{s}$, at which point the satellite looses its mass more rapidly. Here $r_{s}$ is the subclump scale size, i.e. the radius where the logarithmic slope of the profile is $-2, \rho_{c} \equiv 3 H^{2} / 8 \pi G$ is the critical density, and $\delta_{c}$ indicates the characteristic density contrast of the clump. In Figure the radii $r_{s}$ for three NFW tracks are marked with asterisks. If the core of the NFW profile is well below the tidal stripping boundary the subclump can survive in the inner halo without losing much mass. According to the $r_{s}-\delta_{c}$ relation given in Navarro et al. (1997), extrapolated to the mass range relevant for this paper (two examples are shown in the figure), SCDM halos are right near this boundary of destruction and survival at $R \sim 30 \mathrm{kpc}$. Highresolution N-body simulations show, however, a large scatter about this relation (Bullock et al. 1999) and in low density models such as currently popular $\Lambda \mathrm{CDM}$ the halos are more concentrated and thus more able to survive $-r_{s}$ is reduced by a factor of $\sim 0.6$. Since it takes several orbits for a subclump to be completely destroyed even if unstable to tidal disruption, and satellites are being continuously accreted by more massive galaxies, it is expected that a significant population of subclumps will exist within the Einstein radius of the host halo,

$$
\theta_{E} D_{l}=4 \pi\left(\frac{\sigma_{\text {halo }}}{c}\right)^{2} \frac{D_{\mathrm{ls}} D_{l}}{D_{s}} \approx 10 h^{-1} \mathrm{kpc} \frac{H_{0} D_{\mathrm{ls}} D_{l}}{c D_{s}}\left(\frac{\sigma_{\text {halo }}}{156 \mathrm{~km} \mathrm{~s}^{-1}}\right)^{2} .
$$

Here $D_{l}, D_{s}$, and $D_{\mathrm{ls}}$ are the angular size distances to the lens, source, and from the lens to the source, respectively, and $H_{0}=100 h \mathrm{~km} \mathrm{~s}^{-1} \mathrm{Mpc}^{-1}$ is the Hubble constant today. The fraction of mass that remains in subclumps is investigated more fully in section 3 where a specific substructure model is adopted.

Another constraint on subclumps comes from the requirement that they not disrupt the observed population of globular clusters. This is actually more stringent than the constraint imposed by over heating of the Galactic disk, which requires a large density of $m>10^{6} \mathrm{M}_{\odot}$ satellites (Carr \& Lacey 1987; Moore \& 


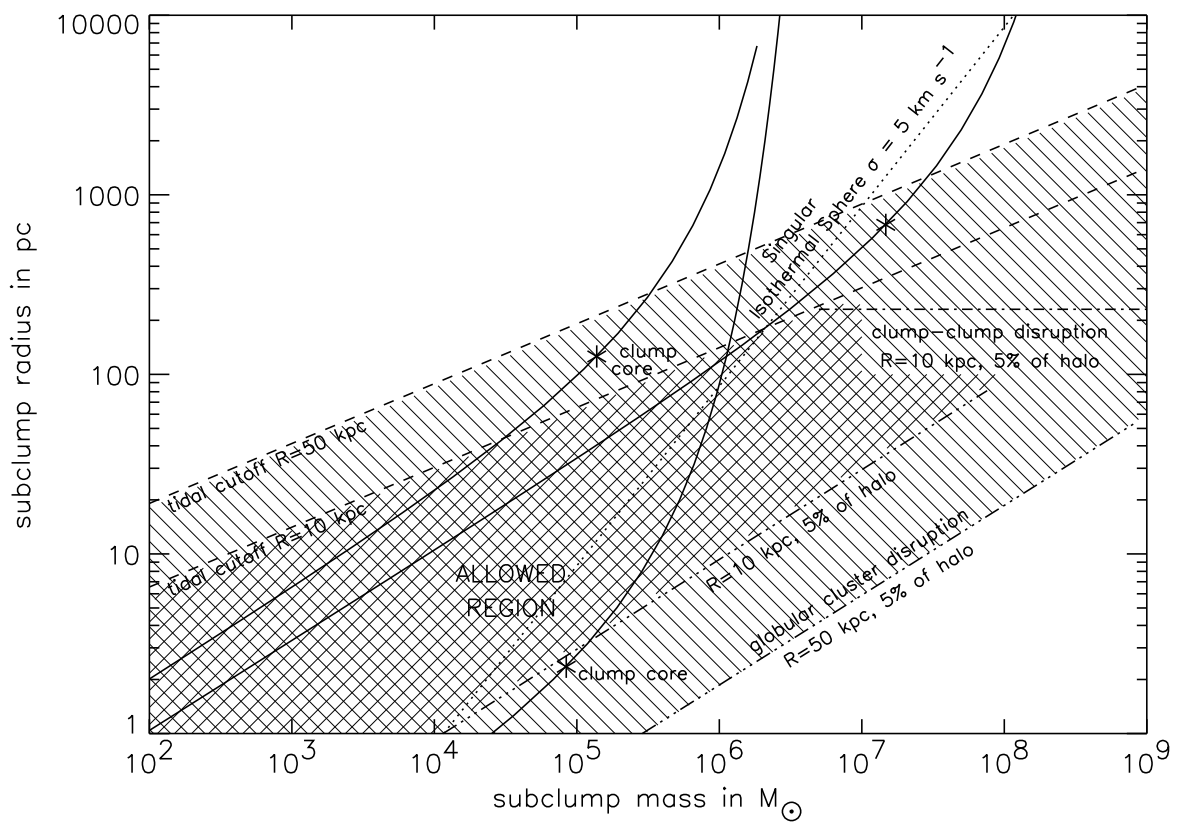

Fig. 1. - Survival of dark matter satellites in a host halo modeled as a singular isothermal sphere of circular velocity $V_{c}=226 \mathrm{~km} \mathrm{~s}^{-1}$. Dashed lines: tidal stripping radius as a function of subclump mass at two different distances from the host halo center, $R=10$ and $50 \mathrm{kpc}$. Dot-dot-dashed lines: constraints imposed by the survival of globular clusters of mass $m_{g}=5 \times 10^{4} \mathrm{M}_{\odot}$ and radius $r_{g}=10 \mathrm{pc}$ for $10 \mathrm{Gyr}$. Dot-dashed line: collisional timescale between subclumps, assuming $t_{\mathrm{ss}}=10 \mathrm{Gyr}$ (see text for details). Dotted curve: $m(r)$ for a SIS with $\sigma=5 \mathrm{~km} \mathrm{~s}^{-1}$. Solid curves: three examples of $m(r)$ for NFW halos which differ in the choice of $r_{s}$ and $\delta_{c}$. The asterisks mark $r_{s}$. The two curves with larger $r_{s}$ follow the $r_{s}-\delta_{c}$ relation given in Navarro et al. (1997) for the $\Omega_{M}=1 \mathrm{SCDM}$ model with $h=0.65$ extrapolated to these masses. In a $\Lambda$ CDM model with $\Omega_{\Lambda}=0.75, r_{s}$ is about 0.6 times smaller for the same clump mass, so satellites will penetrate further into the halo.

Silk 1995). If the subclumps are larger than globular clusters, the disruption timescale $t_{\mathrm{sg}}$ can be estimated in the same way as Spitzer (1958) estimated the timescale for disrupting open star clusters by molecular clouds (Binney \& Tremaine 1987), i.e.

$$
t_{\mathrm{sg}}=\frac{E}{\dot{E}} \sim \frac{0.03 \sigma_{\text {halo }} m_{g} r^{2}}{G r_{g}^{3} m^{2} \eta}
$$

where $\eta$ is the number density of satellites, $\sigma_{\text {halo }}$ is the velocity dispersion in the parent halo, and $m_{g}$ and $r_{g}$ are the mass and radius of the clusters. This constraint is shown in Figure 1 assuming that the fraction of the halo mass in satellites is $5 \%, t_{\mathrm{sg}}=10 \mathrm{Gyr}, m_{g}=5 \times 10^{4} \mathrm{M}_{\odot}$, and $r_{g}=10 \mathrm{pc}$. It sets a limit on the number of dense clumps, not on their size or mass.

Similarly, the subclump-subclump collisional disruption timescale can be estimated by replacing in equation (5) the mass and radius of a typical globular cluster with those of dark matter substructure,

$$
t_{\mathrm{ss}} \sim 0.03 \sigma_{\text {halo }}(G m r \eta)^{-1} .
$$

This is also plotted in Figure 1 for $t_{\mathrm{ss}}=10 \mathrm{Gyr}$. Again, the subclumps do not really need to survive this long. Since cosmological simulations show a continuous replenishment of satellites from infall, their number could remain high even when collisional disruption is actually taking place. The survival of the baryonic 
content of infalling clumps is further complicated by shock stripping. Most of the gas may be removed in this way, making it difficult to interpret any observations of visible matter in terms of dark satellites, for example in the case of the high velocity halo clouds detected in neutral hydrogen (Blitz et al. 1999).

\section{Distribution of satellites}

We seek to develop a simple model for satellites within host halos that incorporates all the aspects of real substructure that are important to lensing. We will use a simple power-law model for the subclumps' internal structure

$$
m\left(m_{a}\right)=m_{a}\left(r / r_{a}\right)^{\beta} .
$$

The parameter $r_{a}$ is kept fixed while the mass within this distance, $m_{a}$, changes for different clumps. Different stages in the stripping of an NFW halo can then be represented by different $\beta$ 's $-\beta=2$ for $r_{t}(R) \lesssim r$ and $\beta \simeq 0.01$ for $r_{t}(R) \gtrsim r$. For the special case of a SIS subclump $\beta=1$ and $m_{a}=2 \sigma^{2} r_{a} / G$. Combining this with the tidal radius (11) gives the mass as a function of galactic radius

$$
m\left(m_{a}, R\right)=\left[\frac{m_{a}^{3 / \beta}}{3 M(R)}\left(\frac{R}{r_{a}}\right)^{3}\right]^{\frac{\beta}{3-\beta}} .
$$

In our model the number density of subclumps, $\eta$, is assumed to be proportional to the average or total mass density - clumped plus smooth components. In this way the clumps flow with the mass. Dynamical friction is taken to be unimportant. The distribution of satellites is modeled as a power-law in $m_{a}$,

$$
\frac{1}{\bar{\rho}} \frac{d \eta}{d m_{a}}\left(m_{a}\right)=\frac{G}{4 m_{0} r_{a} c^{2}}\left(\frac{m_{a}}{m_{a}^{\max }}\right)^{\frac{\beta-3 n}{3-\beta}},
$$

where $\bar{\rho}$ is the total local density and $m_{a}^{\max }$ is the maximum normalized mass. The above is an $R$-independent quantity. The exponent is chosen so that the true mass function is $d \eta / d m \propto m^{-n}$ where the normalization will depend on $R$ and the structure of the host halo. CDM simulations favor $n \simeq 2$.

The minimum normalized mass will be set by the parameter $f_{m} \equiv m_{a}^{\min } / m_{a}^{\max }$ : the requirement that the mass in clumps cannot exceed the total halo mass sets a lower bound on $f_{m}$ if the mass function is steep enough. There is a mass below which satellites do not contribute significantly to the lensing and this cutoff is in practice more restrictive than the former bound. For this reason the lensing properties become insensitive to $f_{m}$ when it is below some level.

With equations (8) and (9) we can easily calculate the mass fraction in subclumps as a function of galactic radius for fixed $\beta$. Integrating this along the line of sight gives the fraction of surface density in substructure, $f_{\Sigma}(R)$. This has been done in figure 2 for a NFW host halo. It can be seen here that for small $\beta$, which approximates the case of NFW subclumps with $r_{s} \lesssim r_{t}(R), f_{\Sigma}(R)$ remains relatively independent of $R$. By the definition of a subclump $r_{s}<R_{s}$ so we expect that substructure will survive relatively intact in the region $R \gtrsim R_{s}$. As $R$ further decreases $f_{\Sigma}(R)$ will slowly decrees until $r_{t} \simeq r_{s}$ at which point it will drop more steeply. It seems realistic that at $R \sim 1 \mathrm{kpc}$, the scale on which multiple QSO images are formed, $f_{\Sigma}(R)$ does not drop below $20 \%$ of the what it is at $R=R_{s}$ ( $\sim 20 \mathrm{kpc}$ for the Milky Way). Numerical simulations find that $10-20 \%$ of the mass within the virial radius of a $10^{12} \mathrm{M}_{\odot}$ halo is contained in substructures of mass $>10^{7} \mathrm{M}_{\odot}$ (Moore et al. 1999a). The lensing properties will be sensitive to structures that are several 


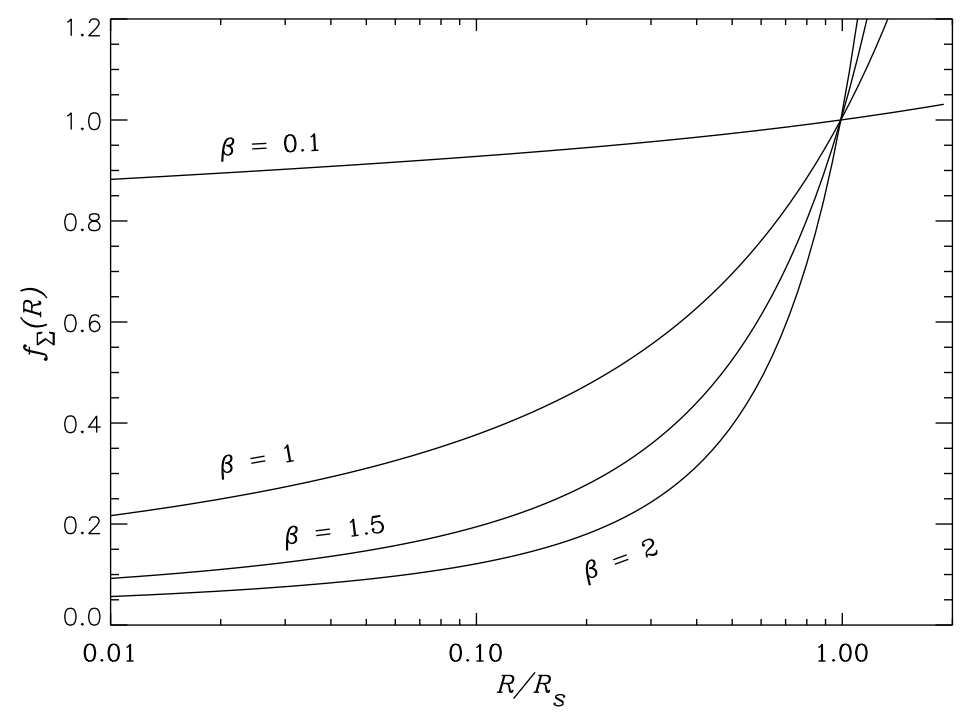

Fig. 2.- The fraction of surface density in substructure as a function of radius from the center of a NFW halo. The scale size of the host halo is $r_{s}=R_{S}$ (see equation 3). This is independent of $\delta_{c}$. In all cases the normalization is chosen so $f_{\Sigma}\left(R_{s}\right)=1$. SIS subclumps are labeled $\beta=1$. The core of NFW profiles is $\beta=2$ and the core of a Moore profile (Moore et al. 1999b) is $\beta=1.5$. For comparison $R_{s}$ is expected to be $\sim 20 \mathrm{kpc}$ for the Milky Way halo.

orders of magnitude smaller - below the resolution of any existing simulation. This simple model strongly supports our conjecture that in the CDM model $f_{\Sigma}(R)$ is greater than a few percent at the point where multiple images are formed and could potentially be much larger. An accurate determination of $f_{\Sigma}(R)$ for the relevant mass range will require CDM simulations with mass resolution $4-5$ orders of magnitude better then those available today.

For the lensing simulations discussed later the subclumps will be modeled as SISs and the distribution will be renormalized to the desired $f_{\Sigma}$. The distributions of magnifications and image distortions will be only weakly dependent on the SIS assumption because the angular sizes of the sources we consider are not small compared to the sizes of the subclumps, as will be seen in section 6.2.2. In this case the magnification averaged over the image will not depend on the details of the subclumps' internal structure. The lensing is more dependent on $f_{\Sigma}$ which makes it a more useful free parameter. For SIS subclumps $m_{a}$ can be replaced by $\sigma$ in the above giving

$$
\begin{aligned}
r_{t}(\sigma, R)= & \sigma \sqrt{\frac{2 R^{3}}{3 G M(R)}} \quad m(\sigma, R)=\sigma^{3} \sqrt{\frac{8 R^{3}}{3 G^{3} M(R)}} \\
& \frac{1}{\bar{\rho}} \frac{d \eta}{d \sigma}(\sigma)=\frac{1}{m_{0} \sigma_{\max }}\left(\frac{\sigma}{\sigma_{\max }}\right)^{2-3 n}
\end{aligned}
$$

\section{Lensing by substructure}

A probe of purely gravitational mass is needed to confirm or disprove the existence of substructure in galaxy halos. We are interested in the situation where there is one galaxy size gravitational lens creating 
multiple images of a background source. Within this lens there is a large number of randomly positioned subclumps that affect each of the images. We will ignore the effects of any additional clumps outside of the main lens. The large number of subclumps and the small, but not vanishingly small, size of the source will require simulations of a small region of the lens plane rather than the entire lens at once. The contribution from the parts of the lens outside the simulation region must therefore be represented in some way. The theoretical background to our method is presented in this section.

If no substructure existed in the lens it would have the surface density $\kappa_{\text {smooth }}\left(\vec{x}^{\prime}\right)$ expressed in units of the critical density, $\Sigma_{c} \equiv c^{2} D_{s}\left(4 \pi G D_{l} D_{\mathrm{ls}}\right)^{-1}$. We will subdivide this density into two components within the region of the lens plane being simulated; the substructure component, $\kappa_{\text {sub }}\left(\vec{x}^{\prime}\right)$, and the halo component,

$$
\kappa_{\text {halo }}\left(\vec{x}^{\prime}\right)=\left\{\begin{array}{lc}
\kappa_{\text {smooth }}\left(\vec{x}^{\prime}\right) & \text { outside region } \\
{\left[1-f_{\Sigma}\left(\vec{x}_{o}\right)\right] \kappa_{\text {smooth }}\left(\vec{x}^{\prime}\right)} & , \quad \text { inside region }
\end{array}\right.
$$

where $\vec{x}_{o}$ is the center of the region. The simulation region is made large enough that, for the purposes of lensing, the mass outside the region can be considered smoothly distributed. It is assumed that $f_{\Sigma}\left(\vec{x}^{\prime}\right)$ does not change over the simulation region.

In the thin lens approximation, the light deflection angle caused by the two surface density components will add. This follows from linear superposition of the surface potentials. The lensing equation can thus be written

$$
\vec{y}^{\prime}=\vec{x}^{\prime}-\vec{\alpha}_{\text {halo }}\left(\vec{x}^{\prime}\right)-\vec{\alpha}_{\text {sub }}\left(\vec{x}^{\prime}\right) .
$$

Here the unlensed angular position of the source, $\overrightarrow{\theta_{s}}$, the position of the image, $\vec{\theta}_{i}$, and the deflection angle, $\vec{\alpha}^{\prime}(\vec{x})$, have been rescaled

$$
\vec{x}^{\prime}=\frac{\overrightarrow{\theta_{i}} D_{l}}{\lambda_{o}} \quad \vec{y}^{\prime}=\frac{\overrightarrow{\theta_{s}} D_{l}}{\lambda_{o}} \quad \vec{\alpha}\left(\vec{x}^{\prime}\right)=\frac{D_{l} D_{l s}}{\lambda_{o} D_{s}} \vec{\alpha}^{\prime}\left(\lambda_{o} \vec{x}\right)
$$

where $\lambda_{o}$ is any arbitrary scale that can be set to a convenient value (usual making $|\alpha(x)| \sim 1$ ). In the absence of substructure the image in question will appear at $\vec{x}_{0}$ :

$$
\vec{y}_{0}=\vec{x}_{0}-\vec{\alpha}_{\text {smooth }}\left(\vec{x}_{0}\right) .
$$

Subtracting equation (15) from (13), and shifting to the coordinates $\vec{x} \equiv \vec{x}^{\prime}-\vec{x}_{0}$ and $\vec{y} \equiv \vec{y}^{\prime}-\vec{y}_{0}$, yields

$$
\vec{y}=\vec{x}-\vec{\alpha}_{\text {halo }}\left(\vec{x}+\vec{x}_{0}\right)+\vec{\alpha}_{\text {smooth }}\left(\vec{x}_{0}\right)-\vec{\alpha}_{\text {sub }}\left(\vec{x}+\vec{x}_{0}\right) .
$$

We need to express $\vec{\alpha}_{\text {halo }}(\vec{x})$ in terms of $\vec{\alpha}_{\text {smooth }}(\vec{x})$. We make the approximation $\kappa_{\text {smooth }}\left(\vec{x}^{\prime}\right)-\kappa_{\text {halo }}\left(\vec{x}^{\prime}\right)=$ $f_{\Sigma} \kappa_{\text {smooth }}\left(\vec{x}_{o}\right)$ inside the simulation region. The constant surface density results in a linear, isotropic deflection term $f_{\Sigma} \kappa_{\text {smooth }}\left(\vec{x}_{o}\right) \vec{x}$ so that the final lensing equation is

$$
\vec{y}=\vec{x}-\vec{\alpha}_{\text {smooth }}\left(\vec{x}+\vec{x}_{0}\right)+\vec{\alpha}_{\text {smooth }}\left(\vec{x}_{0}\right)+f_{\Sigma}\left(\vec{x}_{0}\right) \kappa\left(\vec{x}_{0}\right) \vec{x}-\vec{\alpha}_{\text {sub }}\left(\vec{x}+\vec{x}_{0}\right) .
$$

The magnification matrix, $A_{i j} \equiv \partial y^{i} / \partial x^{j}$, of equation (17) is the true observed magnification matrix. This is the lensing equation used throughout the rest of this paper.

One might be concerned that besides the isotropic linear term, $f_{\Sigma}\left(\vec{x}_{0}\right) \kappa\left(\vec{x}_{0}\right) \vec{x}$, in equation (17) there might also be anisotropic linear, or shear, term of the same order. This is the shear caused by the surface density $f_{\Sigma} \kappa_{\text {smooth }}\left(\vec{x}+\vec{x}_{0}\right)$ inside the simulation region. If the region is small compared to the size of the whole 
halo this shear turns out to be very small. To see this consider a spherically symmetric mass distribution. The contribution to the shear at point $R$ from the mass within the shell $R>r>R-\delta r$ is the total shear, $\gamma(r)$, minus what the shear would be were the shell empty. Since $\gamma(r) \propto r^{-2}$ outside any spherically symmetric mass, and the mass exterior to $R$ does not contribute to $\gamma(R)$, the contribution of this shell is

$$
\gamma_{\text {shell }}=\gamma(R)\left[1-\frac{(R-\delta r)^{2} \gamma(R-\delta r)}{R^{2} \gamma(R)}\right] .
$$

For a singular isothermal sphere $\gamma(r) \propto r^{-1}$, so $\gamma_{\text {shell }} / \gamma(R)=\delta r / R$. As long as the simulation region is small compared to the distance to the center of the host halo the mass exterior to this region will dominate the shear in the smooth halo model. If the halo is asymmetric it is only asymmetries on a scale smaller than the simulation region that could change this conclusion. In all the cases we will consider, the simulation region will be a factor of 100 or more smaller than the distance between the image and the center of the lens, $R$. We can then safely ignore this shear term as is done in equation (17). Another related consideration is that, in the limit where the number of subclumps becomes very large at constant surface density, the distortion of the image should converge to the smooth lens solution. In our simulations the satellites will be distributed uniformly over the region considered so it is incapable of producing a shear in this limit. As a result the extra shear must be zero.

It is instructive at this point to estimate how large of an effect substructure is expected to have. Expanding equation (17) around $\vec{x}_{0}$ we can estimate the distortion of an image along the two eigenvectors of the smooth model's magnification matrix,

$$
\theta_{I} \sim \frac{\theta_{s}+\left\langle\alpha_{\mathrm{sub}}\right\rangle \lambda_{o} / D_{l}}{1-\left(1-f_{\Sigma}\right) \kappa \pm \gamma}
$$

It is apparent that the effects of substructure will be magnified or demagnified by the larger scale lens as represented by the denominator in equation (19). Dark matter subclumps will have a significant effect on the image only when the deflection is a significant fraction of the angular size of the source $\theta_{s} \lesssim\left\langle\alpha_{\text {sub }}\right\rangle \lambda_{o} / D_{l}$. The question of whether the lensing effect of satellites is detectable reduces then to the question of whether deflections of this order are common. Note that the probability of a subclump contributing to the lensing is boosted by a factor equal to the background magnification $\mu^{b}=\left(\left[1-\left(1-f_{\Sigma}\right) \kappa\right]^{2}-\gamma^{2}\right)^{-1}$, which can be very large near a critical curve where the magnification formally diverges. This, combined with the fact that the line of sight already passes through a high density region, result in substructure having a larger effect than might otherwise be expected.

\section{Lensing simulations}

To calculate the distribution of magnifications for a large number of random configurations of satellites an efficient method of simulating the lensing effect is required. The basic approach we use is to calculate the deflection angle, $\vec{\alpha}_{\text {sub }}(\vec{x})$, at every point on a dense grid, and use equation (17) to determine if $\vec{y}$ lies within a specified source. Since surface brightness is conserved the magnification can be calculated by multiplying the area of a grid cell by the number of grid points found to be in images and dividing by the original size of the source. We are considering images that are smaller than the angular size of the host halo, so the grid will cover a small region over which the average density is approximated as constant. Multiple sources can be considered simultaneously as long as it is certain that none of their images are outside of the gridded region. 
Direct summation of the contributions to $\vec{\alpha}_{\mathrm{sub}}(\vec{x})$ from each subclump is ponderously slow (of order $N_{\text {clump }} N_{\text {grid }}$ ). To speed the calculation up the following approach is used. We scale all lengths to a convenient and fixed $\lambda_{o}$. After a random realization of subclump masses and positions is created, $\vec{\alpha}_{\text {sub }}(\vec{x})$ is calculated in two steps. The mass distribution in clumps can be related to the deflection angle through the lensing potential, which can be broken into two parts $\psi(x)=\delta \psi(x)+\psi^{o}(x)$. The background potential, $\psi^{o}(x)$, is the solution to $\nabla^{2} \psi^{o}(\vec{x})=2 \bar{\kappa}$ where $\bar{\kappa}$ is the average surface density of substructure within the simulation region. This is easily solved. The perturbed potential and the deflection angle are then given by

$$
\nabla^{2} \delta \psi(\vec{x})=2[\kappa(\vec{x})-\bar{\kappa}], \quad \vec{\alpha}_{\text {sub }}(\vec{x})=\vec{\nabla} \delta \psi(\vec{x})+\bar{\kappa} \vec{x} .
$$

An estimate of $\vec{\alpha}_{\text {sub }}(\vec{x})$ can be quickly found by moving the mass of each clump to its closest grid point and solving (20) by fast Fourier transform (FFT).

The contribution to $\vec{\alpha}_{\text {sub }}(\vec{x})$ from nearby subclumps is not well represented in this FFT solution because of the interpolation to a grid and the internal structure of a subclump. To refine the calculation a square patch around each clump is considered. The contribution of this satellite to the FFT estimate is subtracted in this patch, and then a more accurate estimate is recalculated. Here we use the fact that the deflection angle for any spherically symmetric lens is particularly simple (Schneider, Ehlers, \& Falco 1992)

$$
\vec{\alpha}(x)=\frac{\bar{m}(x)}{x} \hat{x} \quad \bar{m}(x) \equiv 2 \int_{0}^{x} d x^{\prime} x^{\prime} \kappa\left(x^{\prime}\right) .
$$

The size of the patch around each satellite is adaptively scaled to be proportional to the size of the clump. This approach results in a $\sim N_{\text {grid }} \log N_{\text {grid }}+N_{\text {clump }} \bar{N}_{\text {patch }}$ scaling where $\bar{N}_{\text {patch }}$ is the average number of grid points in the patches. Generally there are more small subclumps than large ones, and this technique is much faster than the direct sum over satellites.

In this way the FFT is used to calculate the long range "forces", and direct summation is used for short range as in a P3M N-body code. The FFT results in the effective mass distribution being periodic on the scale of the box; in practice, however, the mass distribution is always smooth enough on these scales and the artificial periodicity does not affect the lensing.

Note that if $\bar{\kappa}=f_{\Sigma} \kappa_{\text {smooth }}\left(\vec{x}_{0}\right)$, as it is on average, the second term in the expression for $\vec{\alpha}_{\text {sub }}(\vec{x})$ in (20) will cancel the fourth term in equation (17). This will generally be the case when there are large number of subclumps within the simulation region making statistical fluctuations small. This ensures that the results will converge to the smooth lens case when the subclumps get very dense. In the opposite limit where there are no subclumps in the simulation region $\vec{\alpha}_{\text {sub }}(\vec{x})=0$ and equation (17) will give the correct background magnification.

\section{Examples}

A comparison of our simulations with the data requires a specific lensing system to be modeled in detail. An overall smooth lensing potential needs to be constructed before the effects of substructure on each image can be investigated. We will leave a detailed analysis of specific lensing systems for a later paper, and concern ourselves here with representative examples that will illustrate what can be expected. In section 6.1 some general expectations for the SIS model that is used in the simulations will be discussed. Then in section 6.2 the results of numerical simulations are presented and interpreted. 


\subsection{SIS model}

For simplicity, the host halo will be modeled as a SIS. While to model most observed lens systems some ellipticity or external shear must be added to the SIS model, the effects of substructure will not qualitatively change in an asymmetric model.

Two images will then appear at $x=y \pm 1(y<1)$, where the scale used is $\lambda_{o}\left(\sigma_{\text {halo }}\right)=4 \pi \sigma_{\text {halo }}^{2} c^{-2} D_{l} D_{\mathrm{ls}} / D_{s}$. The unperturbed shear and convergence are

$$
\gamma=\kappa=\frac{\lambda_{o}}{2 r}=\frac{1}{2|x|} .
$$

From equation (10), the subclump - also SISs - have sizes and masses

$$
r(\sigma, R)=\frac{R \sigma}{\sqrt{3} \sigma_{\text {halo }}} \quad m(\sigma, R)=\frac{2 R \sigma^{3}}{\sqrt{3} G \sigma_{\text {halo }}} .
$$

The scaled deflection angle caused by a truncated SIS subclump can be calculated using equation (21),

$$
\vec{\alpha}(x)=\frac{2}{\pi}\left(\frac{\sigma}{\sigma_{\max }}\right)^{2}\left\{\begin{array}{cc}
a-\sqrt{a^{2}-1}+\tan ^{-1} \sqrt{a^{2}-1} & , \quad a>1 \\
a & , \quad a<1,
\end{array}\right.
$$

where $a \equiv r(\sigma, R) / x \lambda_{o}\left(\sigma_{\max }\right)$, and $x$ is the distance defined in equation (14) using $\lambda\left(\sigma_{\max }\right)$.

When the impact parameter $y$ is small the images will form at close to the same $R$ on opposite sides of the lens. In section 3 it was shown that if the subclumps are compact enough compared to the host halo their mass distribution will not be a strong function of $R$. Following this argument we will take $f_{\Sigma}$ to be the same for both images in the simulations. We will also replace the mass and radius distributions at a projected distance $x$ with their values at the radius $R=x \lambda_{o}\left(\sigma_{\text {halo }}\right)$. Most of the mass to located near this radius and a more detailed model would not be warranted at this point.

Let us consider what level of substructure will be important for lensing in this model. From $\S$ we know subclumps will have a significant effect on the image when the deflection caused by an individual satellite is a significant fraction of the angular size of the source. We will use the requirement $\theta_{s} \lesssim 10\left\langle\alpha_{\text {sub }}\right\rangle \lambda_{o} / D_{l}$. A characteristic deflection angle is $\left\langle\alpha_{\mathrm{sub}}\right\rangle \lambda_{o} \simeq \lambda_{o}(\sigma)$. We can then put a loose lower limit on the mass of subclumps that can have a significant effect on the lensed image of a source of physical size $l_{s}$,

$$
m_{c} \simeq \frac{2 R \sigma_{c}^{3}}{\sqrt{3} G \sigma_{\text {halo }}} \sim \frac{2 c^{3} R}{\sqrt{3} G \sigma_{\text {halo }}}\left(\frac{l_{s}}{4 \pi D_{\mathrm{ls}}}\right)^{3 / 2} \sim 5 \times 10^{3} \mathrm{M}_{\odot}\left(\frac{h c}{H_{0} D_{\mathrm{ls}}} \frac{l_{s}}{\mathrm{pc}}\right)^{3 / 2} \frac{200 \mathrm{~km} \mathrm{~s}^{-1}}{\sigma_{\text {halo }}} \frac{R}{\mathrm{kpc}},
$$

where the tidal radius (23) was used. As we shall see this estimate is fairly accurate.

If subclumps with $m>m_{c}$ are rare because the mass fraction in this mass range is small, then it will be unlikely for images to be affected. We can introduce a figure of merit that gauges the effectiveness of substructure to influence images by considering the average number of satellites that intersect an image. An idealized circular image will have a radius of $l_{s} D_{l} \sqrt{|\mu|} / D_{s}$ on the lens plane. If we add this radius to the radius of the subclump we can find the area in which a subclump will influence the image. The average number of subclumps in this region is,

$$
F_{\mathrm{sub}}=\pi \int_{m_{c}}^{m_{\max }} d m^{\prime}\left[r\left(m^{\prime}, \kappa\right)+\frac{l_{s} D_{l}}{D_{s}} \sqrt{|\mu|}\right]^{2} \frac{d \eta}{d m}\left(m^{\prime}\right) .
$$




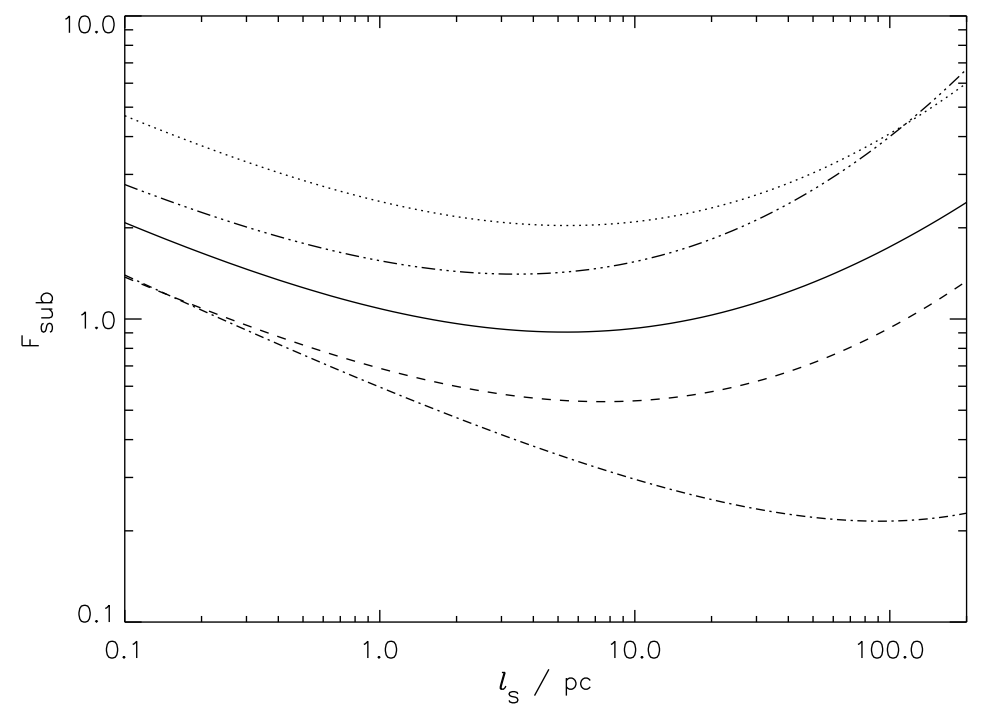

Fig. 3. - Some examples of the substructure lensing parameter $F_{\text {sub }}$ as a function of source size. The solid line is for $\left(f_{\Sigma}, \mu, R, m_{\max }\right)=\left(0.05,9.3,1 \mathrm{kpc}, 10^{10} \mathrm{M}_{\odot}\right)$ where $f_{\Sigma}$ in this case is the fraction of mass in subclumps with $m>m_{c}$. Parameterized in the same way - dotted $\left(0.1,7.3,1 \mathrm{kpc}, 10^{9} \mathrm{M}_{\odot}\right)$, dashed $\left(0.03,5.0,1 \mathrm{kpc}, 10^{9} \mathrm{M}_{\odot}\right)$, dot-dashed $\left(0.03,3.0,5 \mathrm{kpc}, 10^{9} \mathrm{M}_{\odot}\right)$, dot-dot-dashed $\left(0.05,9.3,1 \mathrm{kpc}, 10^{8} \mathrm{M}_{\odot}\right)$. In all cases $z_{s}=3, z_{l}=1$, the subclump mass function is $\propto m^{-2}$, and we assumed a flat cosmology with $\Omega_{M}=1$.

Roughly, if $F_{\text {sub }} \gtrsim 1$ substructure will affect almost every image. How well $F_{\text {sub }}$ characterizes the lensing properties needs to be investigated with numerical simulations, but we can glean some expectations from Figure 3 where $F_{\text {sub }}$ is plotted for some example sets of satellite parameters. Clearly, only a small fraction of the mass needs be in subclumps, $\sim 1 \%$, for them to have a significant influence on images. Generally, when the source is small substructure is important because $m_{c}$ is small and there are many available lenses. As $l_{s}$ increases $F_{\text {sub }}$ decreases until the source gets large enough that it becomes probable for one of the larger satellites to lie close to the image. $F_{\text {sub }}$ would not increase again for large $l_{s}$ as it does in Figure 3 if the mass function were more bottom-heavy than $m^{-2}$. When the grow subclumps larger while keeping their masses fixed the minimum of $F_{\text {sub }}$ is smaller and moves to larger $l_{s}$; the parameter $R$ is used here to normalize the size distribution through equation (23). $F_{\text {sub }}$ does not increase indefinitely with $l_{s}$ because at some point $m_{\max } \simeq m_{c}\left(l_{s}\right)$, and satellites cease to have much of an effect.

The source has been taken to be circular so far, but if the source is highly elongated like a radio jet the likelihood of there being a subclump nearby will be larger. At the same time its small width makes it susceptible to distortion by comparatively small subclumps. The superior resolution of radio interferometers could make these good sources for observing substructure lensing if the surface brightness of the jets is high enough.

Microlensing of multiple image QSOs has already been identified through its time dependence. Stellar mass compact objects have lensing timescales on the order of months. In contrast lensing by substructure will be nearly time independent. The characteristic timescale in this case is $t=\lambda_{o}(\sigma) / v_{\perp}$. This results in

$$
t \simeq 4.1 \times 10^{5} h^{-1}\left(\frac{\sigma}{\mathrm{km} \mathrm{s}^{-1}}\right)^{2}\left(\frac{\mathrm{km} \mathrm{s}^{-1}}{v_{\perp}}\right)\left(\frac{H_{0} D_{l} D_{\mathrm{ls}}}{c D_{s}}\right) \mathrm{yr} .
$$



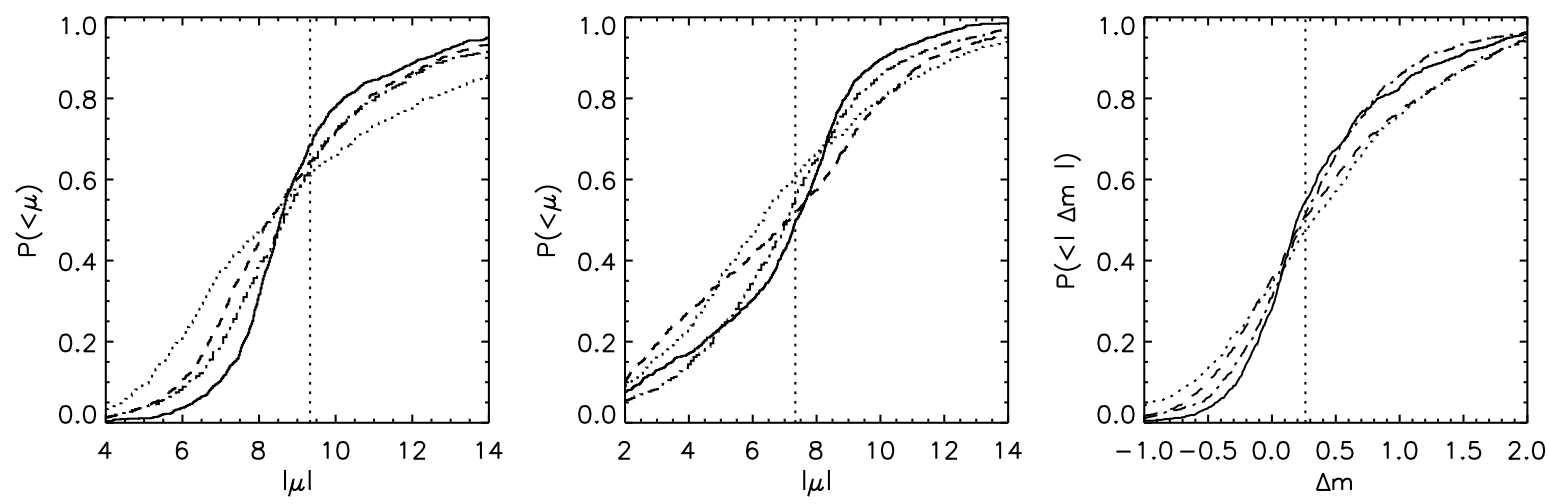

Fig. 4. - The cumulative magnification distributions for the two images of a SIS lens with source position $y=0.12$ measured in lensing scale lengths. The distribution of image 1 - the primary image or outer image - is plotted on the left. In the center is the distribution for image 2. On the right the cumulative distribution of the difference in image brightnesses measured in magnitudes is shown. Negative values of $\Delta m$ correspond to cases where image 2 is brighter than image 1 . The vertical dotted lines are the values expected for a smooth lens and an infinitely small source. The different curves are for different forms of substructure and source sizes. The solid curve assumes that $5 \%$ of the mass is in subclumps with $10^{4} \mathrm{M}_{\odot}<m<10^{8} \mathrm{M}_{\odot}$, and a 10 pc source. Dashed line: same except with $10 \%$ of the mass in substructure. Dash-dotted curve: assumes $5 \%$ of the mass in subclumps with $10^{3} \mathrm{M}_{\odot}<m<10^{7} \mathrm{M}_{\odot}$ and a 1 pc source. Dotted curve: same as the dash-dotted line only with $10 \%$ of the mass in substructure.

For subclumps with $\sigma_{\text {sub }} \sim 10 \mathrm{~km} \mathrm{~s}^{-1}$ and $v_{\perp} \sim 200 \mathrm{~km} \mathrm{~s}^{-1}$ this timescale is very large. It is therefore justified to treat the subclump as stationary.

\subsection{Numerical results}

In the following, different aspects of our simulation results will be discussed which correspond to different methods for probing substructure. The simulations are carried out for a compact $(<1 \mathrm{kpc})$ source at $z=3$ in an Einstein-de Sitter cosmology. The cosmology has little effect on the results. The subclump mass function is $\propto m^{-2}$ in accordance with expectation from CDM models. A $256^{2}$ grid is usually used giving an estimated numerical error in the magnification of $\delta \mu \sim A_{\text {grid }}\left(N^{2} A_{\text {source }}\right)^{-1}=10^{-5} A_{\text {grid }} / A_{\text {source }}$ where the $A$ 's are the area of the grid and source. The grid area changes according to the subclump sizes and the background magnification, but generally an accuracy of $\delta \mu \sim 0.01-0.05$ is attained. Simulations with higher resolution have been run to verify this accuracy estimate.

\subsubsection{Magnifications}

As will be seen in section 6.2 .2 the image positions are not significantly changed by small scale substructure. This allows for the possibility of constructing a smooth lens model from the image positions and perhaps the images of the QSO's host galaxy. The magnification ratios predicted by this model, or family of models, can then be compared with the observations. This will generally require four image systems because two image systems do not contain enough information to constrain the smooth lens model. For simplicity we will investigate a simple SIS lens at $z=1$ with a velocity dispersion of $\sigma_{\text {halo }}=240 \mathrm{~km} \mathrm{~s}^{-1}$; this is a high 

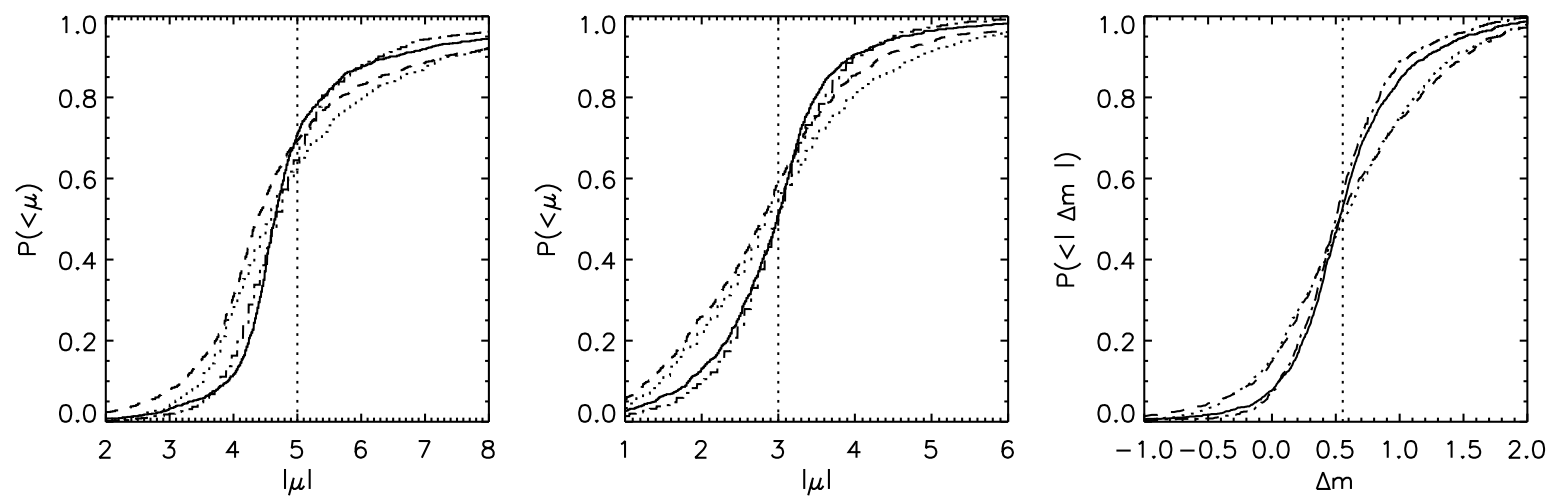

Fig. 5. - This is the same as Figure 4 only the source position is $y=0.25$.
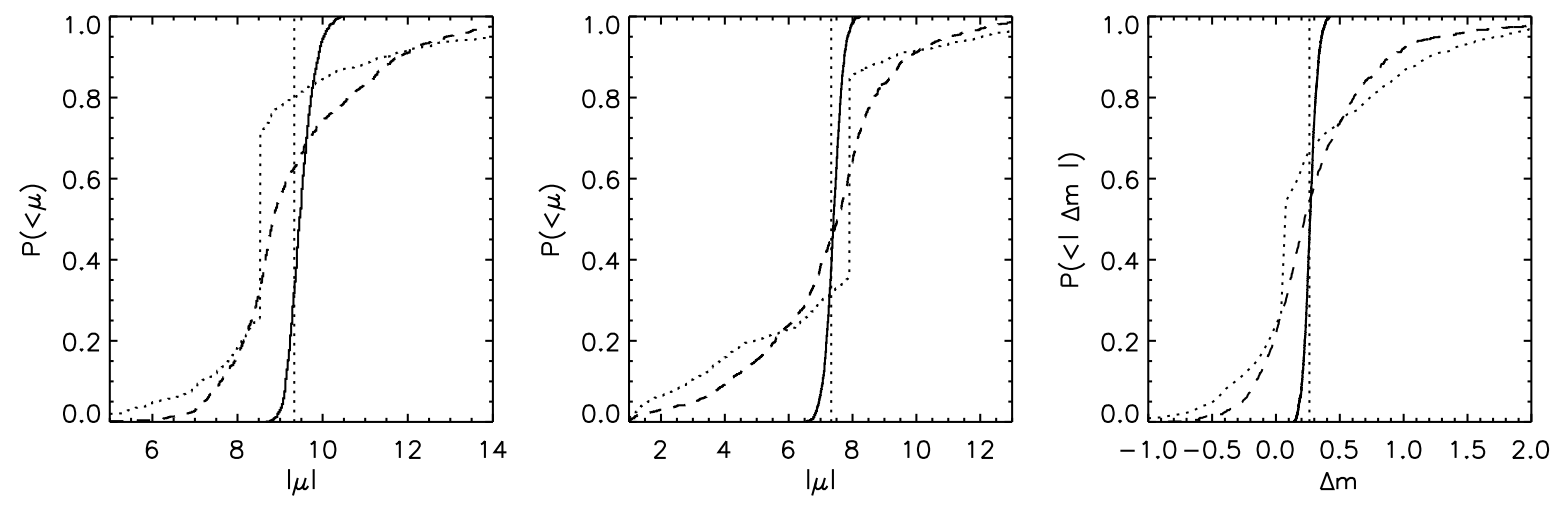

Fig. 6. - The cumulative magnification distributions for the two images of a $10 \mathrm{pc}$ source with source position $y=0.12$. The three different curves correspond to different subclump mass ranges. Solid line: $10^{4} \mathrm{M}_{\odot}<m<10^{5} \mathrm{M}_{\odot}$. Dashed line: $10^{6} \mathrm{M}_{\odot}<m<10^{7} \mathrm{M}_{\odot}$. Dotted line: $10^{7} \mathrm{M}_{\odot}<m<10^{8} \mathrm{M}_{\odot}$. The mass fraction in subclumps, $f_{\Sigma}$, is held fixed at $2 \%$. The subclumps are progressively more influential on the lensing as they get more massive despite their smaller number density. The vertical sections of the dotted curves are located at the background magnifications - the value of the magnification when there are no subclumps influencing the image.

velocity dispersion for galaxies in general, but the population of gravitational lenses tend to be highly biased toward the largest of elliptical galaxies. In any case, if the source position is fixed in units of $\lambda_{o}\left(\sigma_{\text {halo }}\right)$ then $\sigma_{\text {halo }}$ only affects the subclump size distribution - a smaller host halo giving smaller subclumps at the image positions.

The magnification distribution for each image is calculated by repeating the calculation with different realizations of the substructure. Figures 1 and 5 show the cumulative distributions for two choices of the projected source position relative to the center of the host halo. The magnification ratios of the images - the only directly observable quantity - are shown on the right of each figure. This demonstrates that with only a few percent of the surface density contained in substructure the deviations from the expected magnification ratio are significant in most cases. For comparison typical errors quoted for HST observations are $\lesssim 0.05$ mag. For the models in Figures 1 only $14 \%-32 \%$ of the cases, depending on the model, have ratios within $0.2 \mathrm{mag}$ of the expected ratio. For Figures 5 where the source position is further from the lens 

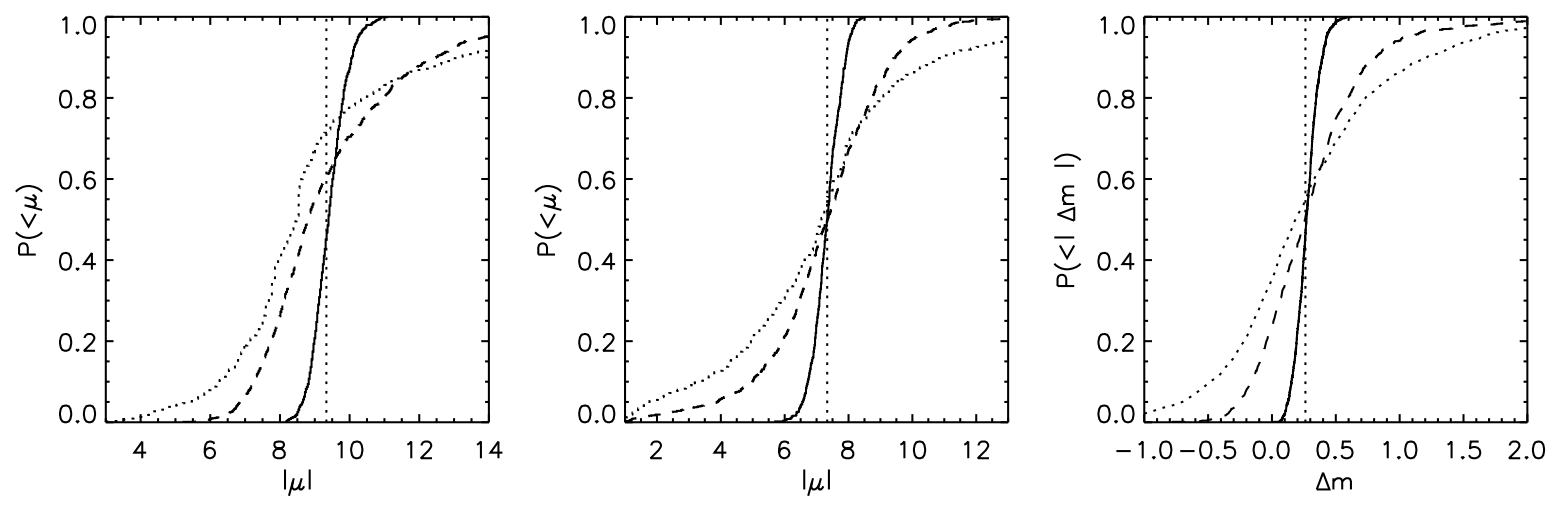

Fig. 7. - Same as in Figure 6 , but the source is $1 \mathrm{pc}$ in size and the mass ranges are: Solid line: $10^{2} \mathrm{M}_{\odot}<m<10^{3} \mathrm{M}_{\odot}$. Dashed line: $10^{4} \mathrm{M}_{\odot}<m<10^{5} \mathrm{M}_{\odot}$. Dotted line: $10^{5} \mathrm{M}_{\odot}<m<10^{6} \mathrm{M}_{\odot}$.

center this is $26 \%-44 \%$ of the cases. For the models in Figure a significant fraction of the cases have the image that is expected to be the brightest instead the dimmer of the two - a sure sign that the lens is more complicated than the lens model reflects.

To gauge the impact of substructure on lensing data consider $n$ lens systems. The likelihood that at least one of the magnification ratios is discrepant by a certain amount, $m$, is $1-p_{1} p_{2} \ldots p_{n}$ where $p_{i}$ is the probability that the $i$ th image pair has a magnification ratio within $m$ of the expected ratio. With only a few systems a strong limit can be put on the amount of substructure as long as the expected magnification ratios can be predicted to a certain degree. For example, if we were to observe 5 independent QSO image pairs like the ones depicted in Figures 1 or 5 , and all of them had ratios within 0.2 mag of the expected values then all the models displayed could be ruled out at better than $98 \%$ confidence.

We expect that if $l_{c}^{3} / \mathrm{m}^{2}$ is held fixed the magnification distribution will be relatively independent of the source size and the subclump mass range; this is because $\lambda_{o}(\sigma) \propto \sigma^{2} \propto m^{2 / 3}$ (see $\S 6.1$ ). A comparison of figures 4 and 1 is generally in agreement with this scaling relation. Without precise knowledge of the source size the strongest constraint would be put on $f_{\Sigma}$. If the substructure masses are reduced while keeping the source size fixed the distribution tends not to extend to as high magnifications because there is an effective limit to how much a small structure can magnify the large sources. For a fixed substructure distribution, as expected from our discussion of $F_{\text {sub }}$ (section 6.1), the images of smaller sources tend to be more strongly affected by substructure. Smaller sources are sensitive to a wider range of subclump masses. Figures 6 and 7 show how the lensing depends on subclump mass. Subclumps of mass $\lesssim 10^{4} \mathrm{M}_{\odot}$ do not have a significant influence on $10 \mathrm{pc}$ sources and $m \lesssim 10^{2} \mathrm{M}_{\odot}$ subclumps do not affect 1 pc sources at the position and redshift considered. This is in general agreement with the estimate of $m_{c}$ made before, equation (25). This is also a useful test of the simulations because as the number density of subclumps goes up the magnification should converge to the smooth lens solution.

For the high substructure mass ranges in Figure 7 and especially Figure 6 there is a bias toward smaller than expected magnification ratios. This is a result of substructure having a different effect on different kinds of images. To understand this consider a small change in the convergence, $\kappa$, (surface density) from the value predicted by the smooth model $\kappa_{o}$. To first order the change in the magnification is

$$
\frac{\Delta \mu}{\mu_{o}} \simeq 2\left(1-\kappa_{o}\right) \mu_{o} \delta \kappa
$$


Clumping increases the probability that an image will land on an underdence region where $\delta \kappa<0$. The primary image - where $\mu_{o}>1$ and $\kappa_{o}<1$ - will have a smaller magnification in this case. For the secondary image, which is reflected with respect to the primary, the situation is more complicated. If $y<0.5$, like it is in our examples, then $\mu_{o}<0$ and $\kappa_{o}<1$, which means the absolute value of the magnification will go up when $\kappa$ goes down. The opposite is true when $y>0.5$ since in this case $\mu_{o}<0$ and $\kappa_{o}>1$ for image two. This biasing when $y<0.5$ does not seem to have a large effect when the mass distribution is steep and extends down to $m_{c}$ as in Figures 1 and 5 . However, if the substructure consists of only high mass objects - relative to the source size - the bias will be significant. This is most noticeable for the $10^{7} \mathrm{M}_{\odot}<m<10^{8} \mathrm{M}_{\odot}$ model in Figure 6 (dotted curves) where in a large fraction of the cases the images have the background magnification, $\mu^{b}$ (the vertical sections of the curves).

When confronting observations the smooth lens model and the substructure model need to be constrained simultaneously in four image lens systems. This task is complicated by degeneracies in smooth lens models which produce equally good fits to the image positions, but different magnification ratios. Constraining the level of substructure in these systems requires significant further development beyond what is presented here and will be the subject of a future paper by one of the authors (Metcalf \& Zhao 2001).

\subsubsection{Image distortions}

Resolution in the visible is not high enough to directly observe distortions to images caused by substructure in the mass range that we are considering here. However radio observations with sub milli-arcsecond resolution could be used to find distortions in one image that are not mirrored in all the other images if the source is of the appropriate size. To investigate this possibility and to gain more understanding of the lensing behavior in general we consider the images produced in the same simulations used in the previous subsection.

Figure 8 shows representations of the images with random realizations of substructure. In most cases the change in the morphology of the image is small. The distortions are typically on milli-arcsecond scales. It can also be seen that the centroid of the image is not significantly shifted. It would take substructures with masses of $\gtrsim 10^{8} \mathrm{M}_{\odot}$ that are very well aligned with the image to change the image position by a few tens of milli-arcsecond. Such a chance alignment will be rare even in the CDM model.

These figures also show that with the sources and substructures we are considering the internal structure of the subclumps should not make a great deal of difference to the lensing. The images feel a deflection potential that is smoothed on the scale of their own size. Since the images are about the same size as the subclumps in these examples how cuspy the subclump cores are will not make a great deal of difference.

Another interesting possibility is a lensed radio jet. Jets typically have opening angles of a few degrees and extend over Mpc (see Kembhavi \& Narlika 1999 and Peterson 1997). This source would then cover more area on the lens plane making it more likely to come close to a large subclump; its thinness would make distortions more pronounced. Figure 9 shows some idealized representations of what a lensed radio jet would look like. Since the jets are not perfectly conical in reality the distortion caused by lensing would need to be deduced by comparing the two images. Some of the distortions are on $\sim 0.01 \operatorname{arcsec}$ scales. There is at least one multiply-imaged radio jet known, Q0957 +561 . The two images have been mapped on milli-arcsecond scales (Gorenstein et al. 1988) and there is not any obvious evidence for substructure in this case although a rather large subclump would be require to produce a noticeable effect $\left(\sim 10^{7} \mathrm{M}_{\odot}\right)$ (Barkana et al. 1999). 


\subsubsection{Distribution of magnification ratios}

Another approach to take in the search for substructure is to look at all multiply-imaged QSOs and consider the overall distribution of magnification ratios without modeling individual cases, only the population of halos in general. This method has several potential advantages besides requiring less work. Two image QSOs can be used in this case, which increases the size of the sample and generally the physical distance the images are from the center of the lens. The latter property means both that substructure will be more likely to survive and that the final constraint will be less dependent on the smooth lens model used. To investigate this situation we fix the source at $z=3$, but choose a random lens redshift and impact parameter according to the assumption of constant comoving density. The physical size distribution of subclumps is kept fixed and is the same as in the previous two subsections. In this case the lensing distribution is independent of the distribution of lens velocity dispersions.

The cumulative distribution of magnification ratios is shown in Figure 10 along with the theoretical prediction for smooth lenses. The most significant effect is an over abundance of cases with magnification ratios close to one and cases where the image that is expected to be brighter is in fact the dimmer image. This is mostly the result of simple spillover caused by extra scatter added to a steep distribution - the biasing discussed in section 6.2.1 does not play a big part. Identifying the image that is expected to be brighter requires some knowledge of the individual lenses. In the simple case of an SIS lens it would just require determining which image is closer to the lens; but without this information - which is often missing in radio surveys - we can still consider the ratio of the dimmer image to the brighter one. This distribution is the dashed one in Figure 10 where it can be seen that there is an overabundance of cases with ratios close to one. A proper comparison with data would require accurate modeling of the selection effects inherent in any lens survey as well as using a more realistic smooth lens model. At this time the publicly available data is very limited and does not put an interesting constraint on substructure. This may change in the near future with the completion of several large, systematic QSO surveys.

\section{Discussion}

We have shown in this Paper that, if dark matter substructure exists in the halos of galaxies and can survive to constitute a percent or more of the surface density at small impact parameters (several kpc) - a possibility that is in general agreement with CDM hierarchical cosmologies, it will cause significant changes to the magnification ratios of multiply-imaged QSOs. Furthermore, if the magnification ratios of just a few QSO image pairs can be predicted from the image positions and other information to an accuracy of a few tenths of a magnitude the existence of such substructure can be confirmed or invalidated. This would allow us to probe primordial structure formation on the scales of $10^{3}-10^{8} \mathrm{M}_{\odot}$. To confront observations more detailed modeling of individual QSO lens systems will be needed. This is the topic of another paper (Metcalf \& Zhao 2001, in preparation).

Besides lens modeling there are several other complicating factors involved in measuring the magnification ratios. A correction for differential extinction must be included in the visible bands. Falco et al. (1999) find a median differential extinction between lensed images of $\Delta E(U-V)=0.04-0.06$ mag so this is not likely to be a large impediment. Another issue is intrinsic variation in the source. If the source is variable and the time delay is not well known it is difficult to interpret the flux ratios. Radio observations avoid the complication of differential extinction, but radio galaxies are often variable. Also the lens galaxy is usually not detected in the radio, making the constraints on the smooth lens model weaker. A combination 
of observations at different wavelengths will be required.

Microlensing by stars in the lens galaxy is also a possible contaminating factor if the source is too small. The continuum emission region of a QSO is believed to be less then $\sim 100 \mathrm{au}$, and is observed to be microlensed in some cases. To avoid this a larger source must be used. The broad line emission region is believed to be about a pc in size, which would make it sensitive the subclump masses $\gtrsim 10^{3} \mathrm{M}_{\odot}$. There are also features in the radio emission that are both smaller and larger than this. They would generally need to be resolved in order to confirm their scale. QSO narrow line regions probably have sizes of $\gtrsim 100 \mathrm{pc}$. The molecular line emission region of AGN is believed to be $1-100 \mathrm{kpc}$ in size, but it is not certain if the $\mathrm{CO}$ emission from QSOs, for example, comes from a localized region or from the host galaxy as a whole (see Kembhavi \& Narlika 1999 for a review of QSO properties). Conclusions drawn from lensing on the existence and mass distribution of substructure will thus depend on the kind of observations used and our knowledge of the sources' internal structure.

Finally, the images could also be lensed by more familiar galactic substructures such as spiral arms (Mao \& Schneider 1998). These are expected to interfere with the constraints on dark matter structure at a small level. The Milky Way disk has a surface density of $\sim 50 \mathrm{M}_{\odot} / \mathrm{pc}^{2}$ and spiral arms are believed to have an amplitude of about $20 \%$ in mass. For a lens at $z=1$ and a source at $z=3$ in an Einstein-de Sitter universe this corresponds to $\kappa=0.003 h^{-1}$. We can use equation (28) to calculate the change this would cause in the magnification, which is $\sim 0.05$ mag in the case of the most magnified image discussed in section 6 . The dark matter subclumps considered here have more influence on the magnification because of their comparatively high mass density. Globular clusters are unlikely to cause significant changes in the magnifications because they do not contain a significant fraction of the halo mass $\left(\sim 10^{-4}\right.$ of the Milky Way halo) as pointed out by Mao \& Schneider (1998).

R.B.M. would like to thank H. Zhao for many constructive discussions. We would like to thank the anonymous referee for constructive criticism. Support for this work was provided by NASA through ATP grant NAG54236 and grant AR-06337.10-94A from the Space Telescope Science Institute (P.M.).

\section{REFERENCES}

Barkana, R., Lehár, J., Falco, E. E., Grogin, N. A., Keeton, C. R., Shapiro, I. I. 1999, ApJ, 520, 479

Binney, J. \& Tremaine, S. 1987, Galactic Dynamics (Princeton, New Jersey: Princeton University Press)

Blitz, L., Spergel, D. N., Teuben, P. J., Hartmann, D., \& Burton, W. B. 1999, ApJ, 514, 818

Bode, P., Ostriker, J. P., \& Turok, N. 2000, preprint (astro-ph/0010389)

Bullock, J., Kolatt, T., Sigad, Y., Somerville, R., Kravtsov, A., Klypin, A., Primack, J., \& Dekel, A. 1999, astro-ph/9908159

Bullock, J. S., Kravtsov, A. V., \& Weinberg, D. H. 2000, ApJ, 539, 517

Carr, B. J. \& Lacey, C. G. 1987, ApJ, 316, 23.

Colin, P., Avila-Reese, V., \& Valenzuela, O. 2000, ApJ, 542, 622

Dekel, A. \& Silk, J. 1986, ApJ, 303, 39 
Falco, E.E., Impey, C.D., Kochanek, C.S., Lehár, J., McLeod, B.A., Rix, H., Keeton, C.R., Muñoz, J.A. \& Peng, C.Y. 1999, ApJ, 523617.

Flores, R. A., \& Primack, J. R. 1996, ApJ, 457, L5

Ghigna, S., Moore, B., Governato, F., Lake, G., Quinn, T., \& Stadel, J. 1998, MNRAS, 300, 146

Gorenstein, M. V., Cohen, N. L., Shapiro, I. I., Rogers, A. E. E., Bonometti, R. J., Falco, E. E., Bartel, N. \& Marcaide, J. M. 1988, ApJ, 334, 42

Johnston, K. V., Spergel, D. N., \& Hernquist, L. 1995, ApJ, 451, 598

Kamionkowski, M. \& Liddle, A. 2000, PRL, 84, 4525

Keeton, C. R., \& Madau, P. 2001, ApJ, 549, L25

Kembhavi, A.K., \& Narlika, J.V., 1999, Quasars and Active Galactic Nuclei: An Introduction (Cambridge University Press)

Klypin, A., Gottlöber, S., Kravtsov, A. V., \& Khokhlov, A. M. 1999a, ApJ, 516, 530

Klypin, A., Kravtsov, A. V., Bullock, J. S., \& Primack, J. R. 2001, ApJ, in press astro-ph/0006343

Klypin, A., Kravtsov, A. V., Valenzuela, O., \& Prada, F. 1999b, ApJ, 522, 82

Li, L.-X., \& Ostriker, J. P. 2001, preprint (astro-ph/0010432)

Mao, S., \& Schneider, P. 1998, MNRAS, 295, 587

Mateo, M. 1998, ARAA, 36, 435

Moore, B. 2001, in 20th Texas Symposium on Relativistic Astrophysics and Cosmology (AIP Conf. Series), in press astro-ph/0103100

Moore, B., Ghigna, S., Governato, F., Lake, G., Quinn, T., Stadel, J., \& Tozzi, P. 1999a, ApJ, 524, L19

Moore, B., Katz, N., \& Lake, G. 1996, ApJ, 457, 455

Moore, B., Quinn, T., Governato, F., Stadel, J., \& Lake, G. 1999b, MNRAS, 310, 1147

Moore, B. and Silk, J. 1995, ApJ, 442, L5.

Navarro, J. F., Frenk, C. S., \& White, S. D. M. 1997, ApJ, 490, 493

Peterson, B.M., 1997, An Introduction to Active Galaxtic Nuclei (Cambridge University Press)

Schneider, P., Ehlers, J., \& Falco, E. E. 1992, Gravitational Lenses (Springer-Verlag)

Sellwood, J., \& Kosowsky, A. 2001, in Gas and Galaxy Evolution (ASP Conf. Series), in press astro$\mathrm{ph} / 0009074$ )

Spitzer, L. J. 1958, ApJ, 127, 17

Tormen, G., Diaferio, A., \& Syer, D. 1998, MNRAS, 299, 728

van den Bosch, F. C., \& Swaters, R. A. 2001, MNRAS, in press astro-ph/0006048 

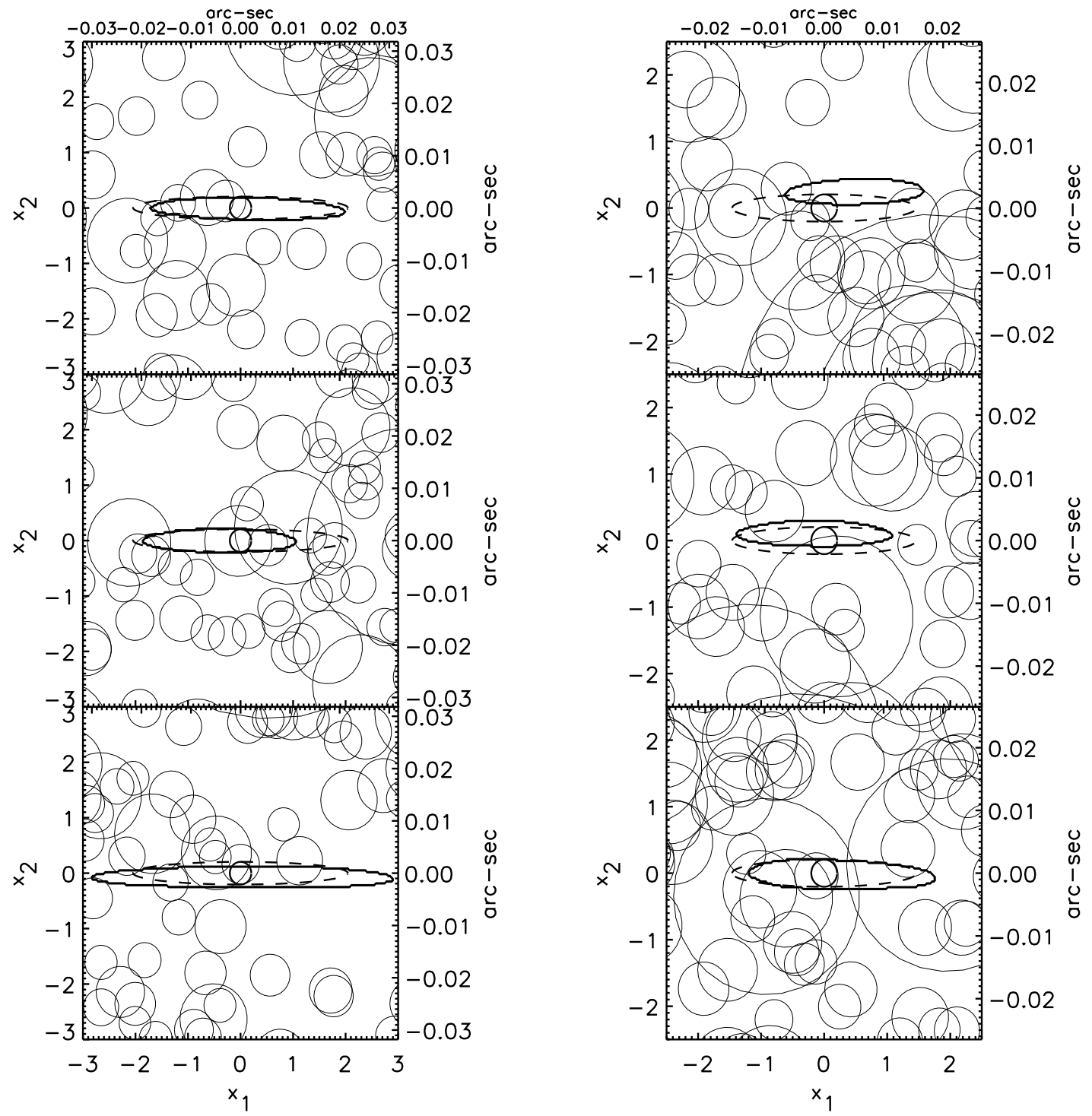

Fig. 8. - Images of a $10 \mathrm{pc}$ source at $z=3$ seen through a lens at $z=1$. On the left are realizations of the outer, primary image and on the right simulations of the inner image. The source position is $y=0.12$. The subclumps constitute $5 \%$ of the density and $10^{4} \mathrm{M}_{\odot}<m<10^{8} \mathrm{M}_{\odot}$ with a $m^{-2}$ mass function. For clarity only a small part of the field used in the simulations is shown and the subclumps with $r<0.3 \lambda_{o}\left(m_{\max }\right)$ are not drawn. This corresponds to masses below $2.0 \times 10^{4} \mathrm{M}_{\odot}$. The radii of the circles are the tidal radii of the subclumps. The thick dashed curves are the shape the image would be if the lens were smooth and the thick circles are what the source would look like if there were no lensing. 

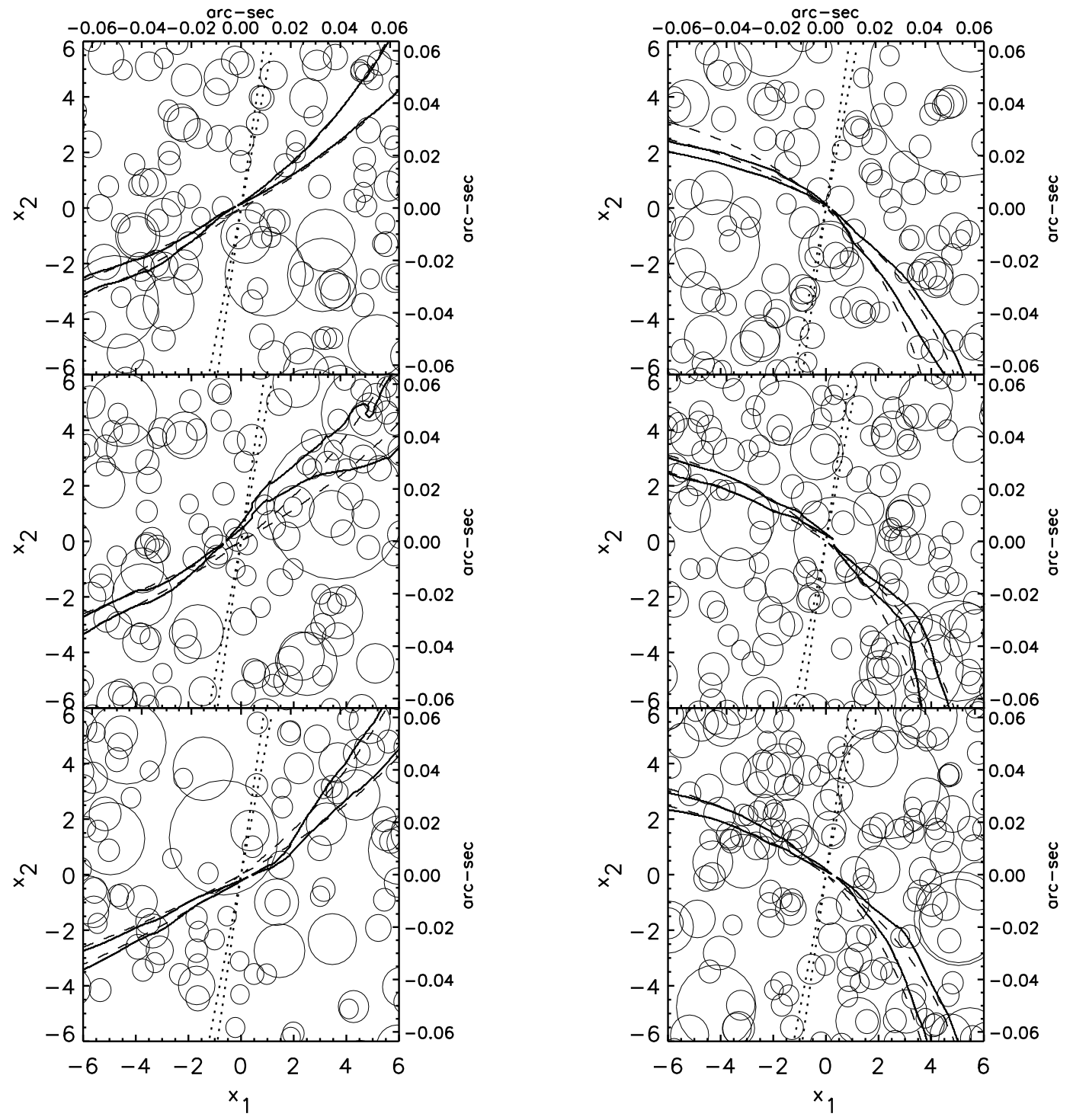

Fig. 9. - Images of an idealized radio jet at $z=3$ seen through a lens at $z=1$. The source of the jet is at $y=0.12$. The subclumps constitute $5 \%$ of the density and $10^{4} \mathrm{M}_{\odot}<m<10^{8} \mathrm{M}_{\odot}$ with a $m^{-2}$ mass function. Only subclumps with $r>0.35 \lambda_{o}\left(m_{\max }\right)\left(m>3.2 \times 10^{4} \mathrm{M}_{\odot}\right)$ are shown. The unlensed jet is shown in dotted lines. The opening angle is $6^{o}$ and it is oriented at $10^{\circ}$ from the lens' radial direction that is the vertical axis here. The dashed lines show the image were the lens smooth. 


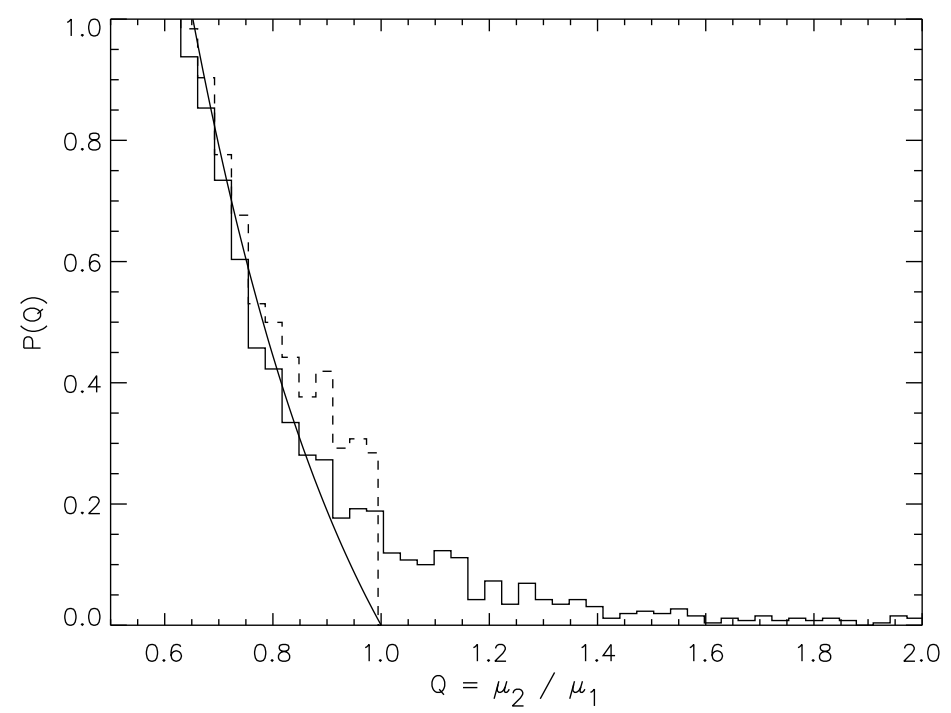

Fig. 10. - The distribution of magnification ratios for a $10 \mathrm{pc}$ source at $z=3$. The smooth curve is for lenses without substructure. The solid histogram includes the effect of subclumps. The dashed histogram is the same except that $Q$ is the lesser of $\mu_{1} / \mu_{2}$ and $\mu_{2} / \mu_{1}$. The subclumps are a fixed $5 \%$ of the lens surface density, and have $10^{4} \mathrm{M}_{\odot}<m<10^{8} \mathrm{M}_{\odot}$. All three distributions have the same integral for $Q>2 / 7$. This cutoff is necessary because the Monte Carlo calculation cannot be efficiently extended down to $Q=0$. However, small $Q$ pairs are strongly selected against in observations and the distribution is not strongly affected by substructure in this region. There are 10,000 realizations in the simulated distribution. 